\title{
Modeling of Target Audience'S Intellectual Behavior
}

\author{
Vitaliy Katsalap
}

How to cite this paper: Katsalap, K.; Modeling of Target Audience'S Intellectual Behavior. Universal Jour nal of Social Sciences and Humanities. 2021, 1, 1 .

https://doi.org/10.31586/ujssh.2021.0 10101

Received: April 06, 2021 Accepted: May 18, 2021 Published: May 20, 2021

Copyright: (c) 2021 by the authors. Submitted for possible open access publication under the terms and conditions of the Creative Commons Attribution (CC BY) license (http://creativecommons.org/licenses /by/4.0/).
Department of Information Security and Information Technologies Employment, The National Defence University of Ukraine, Povitroflotsky Ave. 28, 03049, Kyiv-049, Ukraine.

*Correspondence: typimicku@i.ua

\begin{abstract}
The article considers the problems of theoretical substantiation of negative informational and psychological impact evaluation principles, and conducting of relevant researches in this field. At the same time, due to imperfections of theory and practice of negative informational and psychological impact evaluation, previous researches were conducted mainly by "mirroring" the ways of information counteraction, both for our own measures of information and psychological influence, and for the adversary. The rationale for the methodological approaches used in the organization of information and psychological influence is due to inability to access the adversary's target audiences freely and gather the necessary information, as well as the inadequacy of assessing the degree of change in their behaviour. Characteristics mentioned in article classify target audiences according to defined indicators of remote information control of social and individual behaviour of possible objects of influence. Using the formulated conditions and relying on the psychological and psychophysiological characteristics of individuals, a database of target audience behaviour vulnerabilities is forming, that reflects the dependence of indicators of social behaviour perception changes on the intensity of external negative informational and psychological influences. The algorithm of target audience's information perception is developed based on the model of planned behaviour, in which the subject's overestimation of small values of probabilities are compared in dynamic with decrease of big ones. The proposed algorithm of evaluation of information-psychological impact allows to receive initial data based on which the model of target audience behaviour will be designed in any environmental conditions.
\end{abstract}

Keywords: Information operations; Psychological operations; Social behaviour; Psychological influence; Target audience

\section{Introduction}

According to the main thesis of the Doctrine of Information Security of Ukraine, one of the main ways of its implementation is "development of methodological approaches of negative information and psychological impact assessing". The growth of the Defence Forces of Ukraine requires the implementation of universal planning mechanisms for the entire information security system. First of all - the procedures for negative information and psychological impact identification, that would allow to synchronize information counteraction of all components of the Security and Defence Sector of Ukraine.

The majority of the target audiences think rationally and use the information available to anticipate the consequences of their actions before they decide to do something. After analysing all previous studies [1-3], it became necessary to justify a model that could, to some extent, predict and explain target audiences' behaviours and social attitudes.

Analysis of "Theory of Reasoned Action" showed that its basis corresponds to the research objective and, unlike the "Theory of Perspectives" used by the authors [4] in assessing the probabilities of social group behaviour depend on content of information influence, can be used in substantiating the main indicators of assessing the target audiences' 
future behaviour with the ability to identify relationships between relevant social settings. Particular thing in evaluating the target audiences is its behavioural intentions. The intentions are shaped not only by the attitudes, but also by the influence of certain social norms, which the target audience adheres to.

\section{Formulation of the problem.}

One of the basic principles of assessing negative information and psychological impact is taking into account the predicted behavior of target audience, which does not have or does not perceive responsibility of how to behave. As a result, the target audience's behavior depends on the situation in which it is. This condition shapes behavioral intentions and directly affects the behavior itself - by how the target audience perceives control over own behavioral.

\section{Analysis of recent researches and publications.}

The analysis of the published works shows that today the theory of information security is still imperfect, it lacks clear formal methods and techniques for quantitative assessments of the negative information and psychological impact on certain target audiences. Because of that, assessment of possible target audience's behavioral model, particularly which was formed by negative information and psychological impact, is a difficult task that needs to be addressed.

Based on this, the purpose of the article is to model the intellectual behavior of target audiences.

\section{Outline of the main research material}

Scientific experience shows that the most relevant models of information security research, including the evaluation of negative information and psychological impact, are the Theory of Reasoned Action and The Theory of Planned Behavior.

The Theory of Planned Behavior was developed in 1985-1987 by American scientist Icek Aizen, professor of psychology at the University of Massachusetts, together with Martin Fishbein, professor of psychology at the Institute for Communication Studies at the University of Illinois. The Theory of Planned Behavior is a further development of the Theory of Reasoned Action (TRA), which appeared in 1967 and in eight next years was refined and expanded by the authors mentioned above.

The basis of these theories served many studies in the field of social psychology of the late XIX - first half of XX centuries, which resulted the conclusions about the determined influence of the social settings (attitudes) to human behavior [4].

I. Aizen and M. Fishbein have assumed that majority of people think rationally, and use the information available to anticipate the consequences of their actions before they decide to do something. After analyzing all previous researches, they developed a model that could, to some extent, predict and explain human behavior in relationship with social attitudes. The Theory of Reasoned Action, unlike previous theories, considers the main indicators of future human behavior not solely social attitudes, but also behavioral intentions, formed both: by attitudes, and by the influence of existing social norms that a person adheres to (Figure 1). 


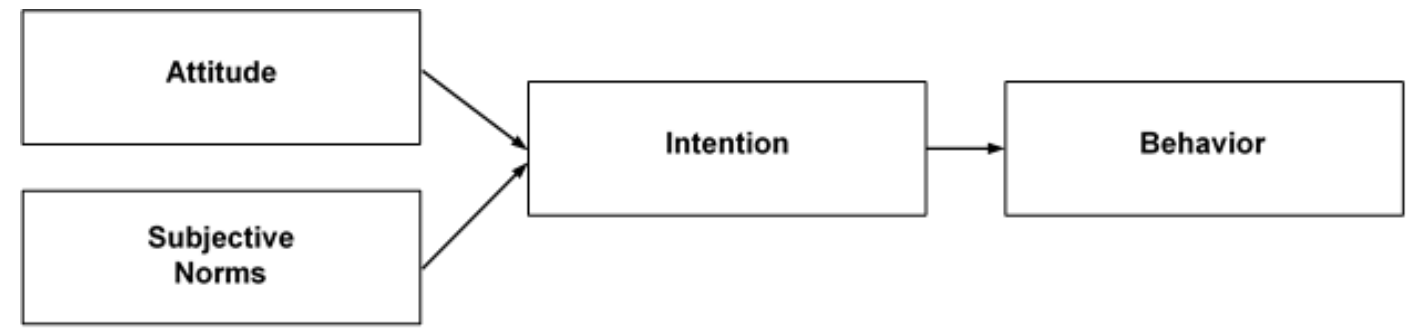

Figure 1. Theory of Reasoned Action Model

This model shows the role of intention in possible target audience's behavior, but the main condition that shapes the information and psychological influence are subjective norms $[5,6]$.

Further studies have shown that this model has drawbacks in predicting the behavior of people who do not have or do not feel responsibility of how to behave. As a result of the work, a third element was added to the model, which forms behavioral intentions and directly influences the behavior itself - perceived behavioral control. The model, thus changing its structure, was called "The Theory of Planned Behavior" (Figure 2).

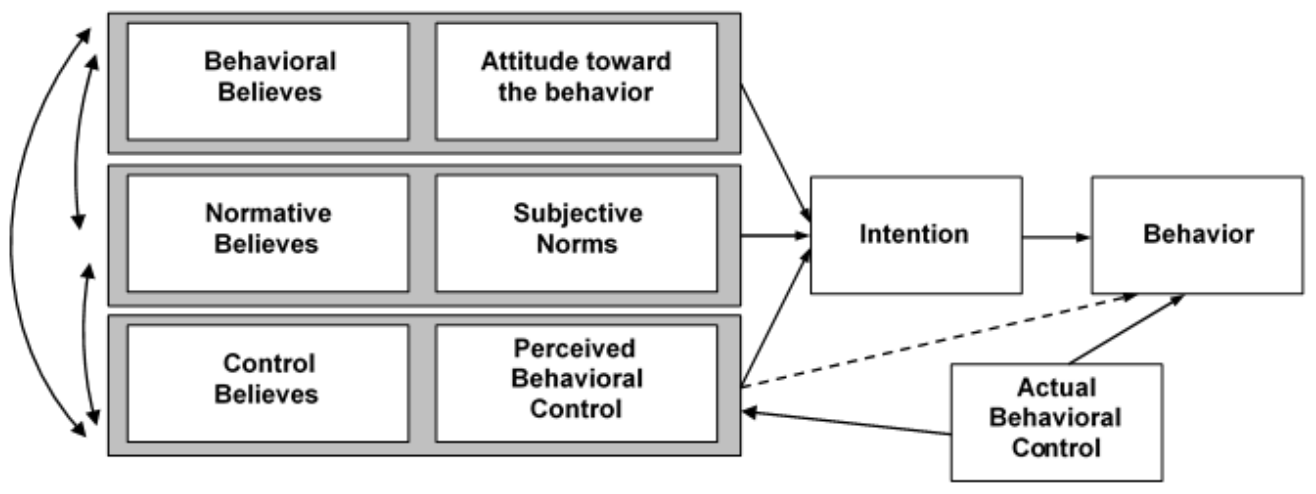

Figure 2. Theory of Planned Behavior Model

If a person perceives the result from their behavior to be positive, he or she will have a positive attitude towards its realization. And vice versa.

If other people, whose opinion is important to that person, evaluate the behavior positively, and person is motivated to meet their expectations, then the subjective norm will be positive. If others evaluate the behavior negatively, and the person seeks to satisfy any of own activities, the result is most likely to be characterized by a negative subjective norm.

In case the behavior is not completely dependents on the will of the person, even if the person may be highly motivated by own attitudes and subjective norms, the behavior may not be performed in the intended way.

Thus, perceived behavioral control shows that person's motivation depends on his or her perception of how well he or she can act in certain conditions.

If person has strong beliefs about the factors positively contributing to the behavior, he / she will have a high degree of perceived behavioral control. Conversely, a person will have a low perception of control if he / she has strong beliefs that impede own behavior. Such perceptions may be based on past experience, predictions of inevitable 
circumstances, attitudes of influential persons whose opinions are important to the individual.

This theory was later incorporated into the curriculum of education and training courses on psychological operations taught at US military institutions and from 2014 into NATO's training manuals on psychological operations (NATO PSYOPS Handbook). At the same time, with aim of clarity and practical usage, the general scheme of the model was supplemented with additional elements, including personal moods and stimulus (Figure 3).

The third behavioral model proposed for consideration is the Hierarchy of Psychological Effects Model. The first prototype of such model was developed in the late XIX century as a communication model used to stimulate behavior change. It includes three elements: cognition (C), affect (A), and behavior (B), and is called the CAB model. The $\mathrm{CAB}$ model shows the cause-effect relationships, successively from cognition to influence and from influence to behavior. The stage of cognition is what the target audience needs to know or perceive, the stage of influence is what the target audience needs to feel or what to believe, and the stage of behavior is what the target audience needs to do or not do [3].

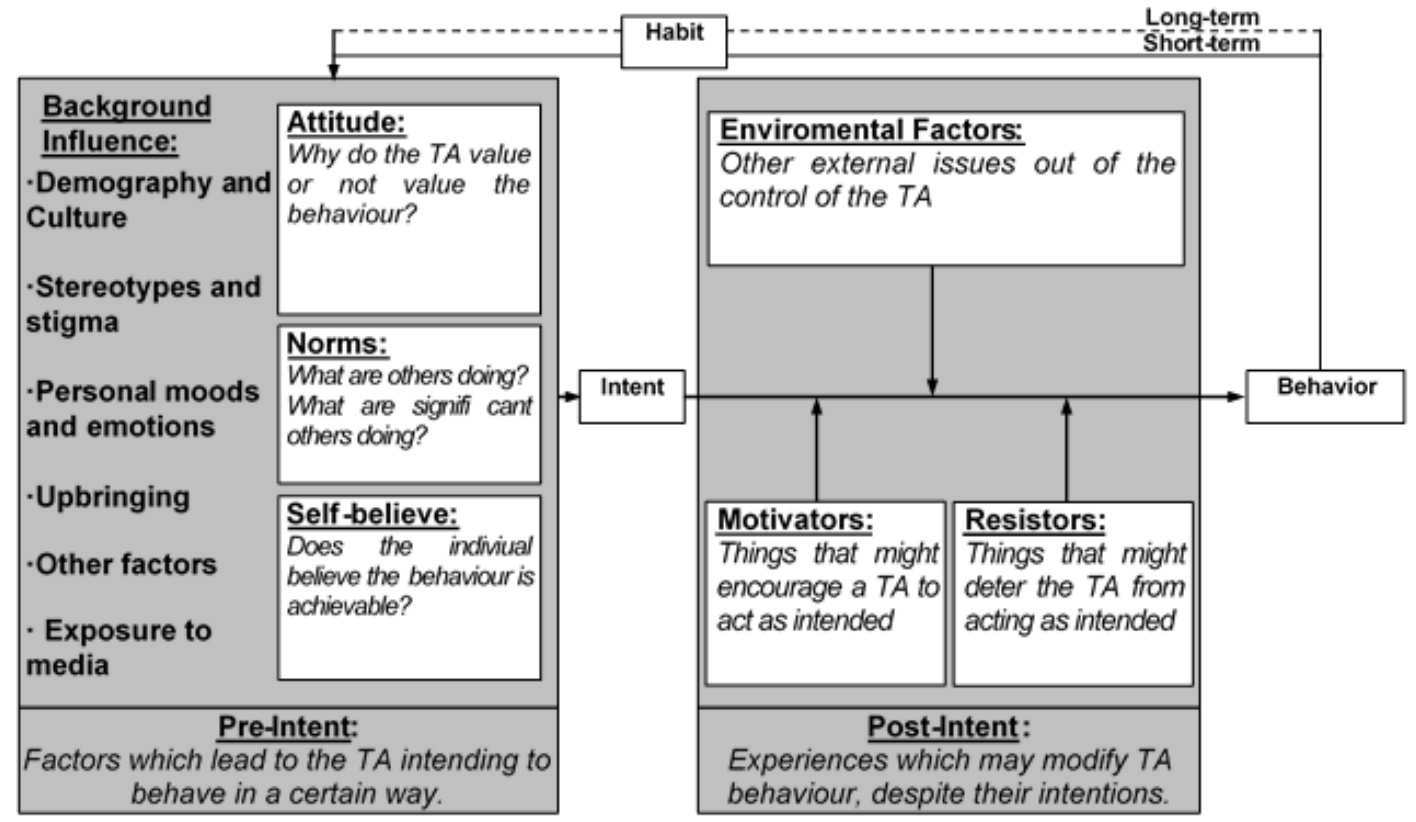

Figure 3. Theory of Planned Behavior Model

The CAB model was further adapted to the needs of planning a psychological operation and expanded from 3 to 6 stages, in order to increase the range of main themes of psychological operations series, namely: awareness, understanding, response, preference, intention and behavior. Influence begins with awareness and understanding because the target audience must perceive the message first before act. The target audience actions are also related to emotions, because of feelings that arise from perception and influence the target audience's desires to respond the message and to behave respectively (Figure 4).

The Hierarchy of Psychological Effects Model helps to develop a complex of psychological operations series (sets of messaging activities, and lethal / non-lethal actions of forces) to achieve consistent intermediate goals from initial to desired behavior of target audience. So, even in case of change of command or units responsible for psychological 
operation, the further activities will continue in previously defined direction. In addition, at the initial stage this model helps in determination the indicators and criteria for evaluating the effectiveness of psychological operation, and after completion this operation - in evaluating the results achieved.

Making certain decision, the target audience builds own behavioral model. This model is needed by the target audience to evaluate the consequences of its decisions.

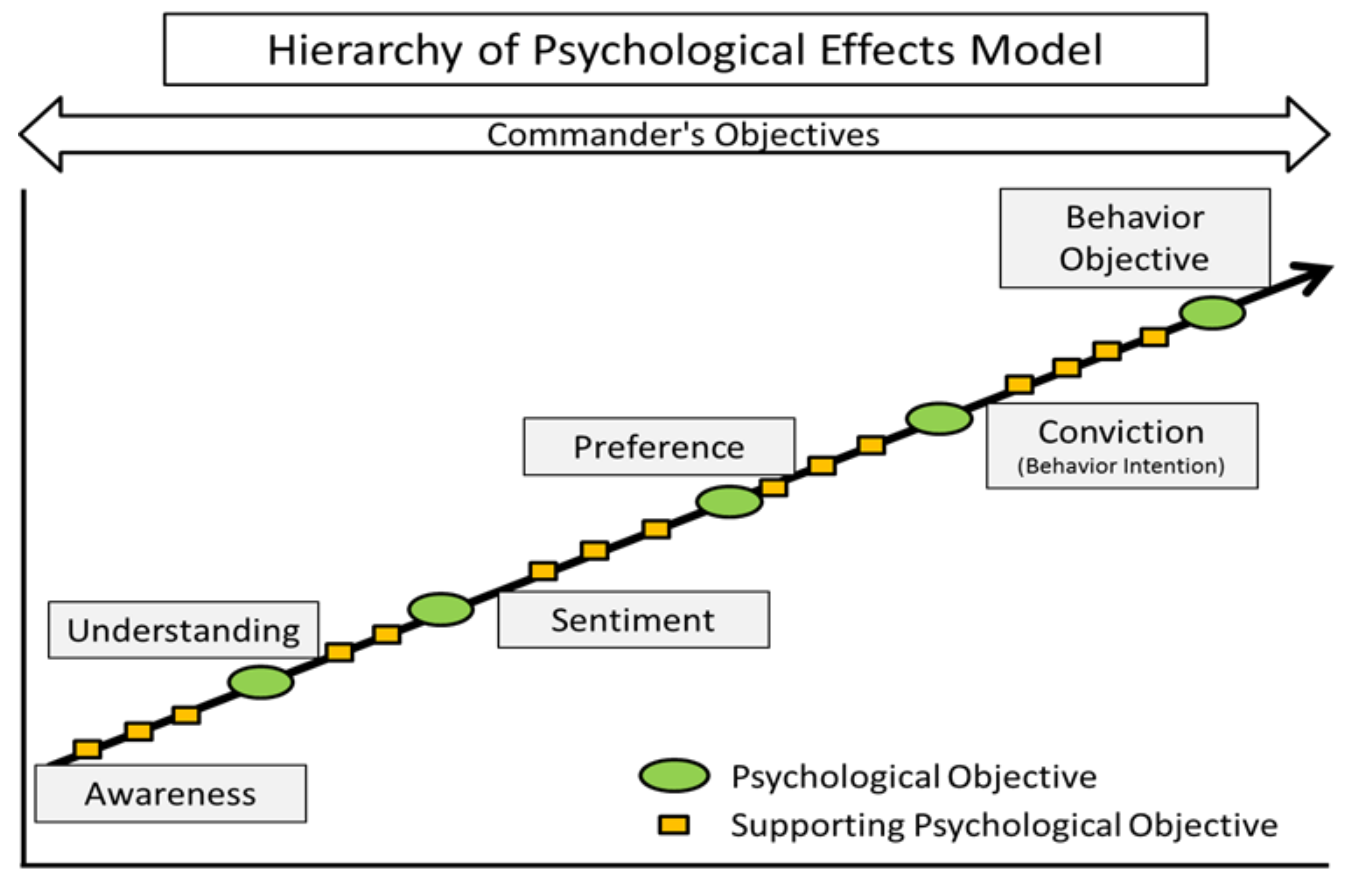

Continuum of Psychological Effects Towards Behavior Objective

Figure 4. Hierarchy of Psychological Effects Model

To formalize the behavioral model, lets consider a set of continuous limited indicators, expressed in a quantitative scale: the likelihood of negative consequences from particular behavior; the expected results or motives; and the degree of importance of certain priorities.

These indicators are divided into two types: probabilistic (the probability of negative consequences occurrence; and degree the goal importance) and interval (the expected result from behavior; weight of motives and priorities; and conditions in which the target audience exists).

The matter of evaluating of negative psychological effect on the specific target audience consists in a clear formalization of behavioral model. Consider the possible conditions that will determine the intended behavior of the target audience.

The condition of social behavior is considered, if the target audience is prone to aroused reaction to external negative psychological influences, which in content, as a rule, aimed at specific behavioral models:

- Motivational (beliefs, values, inclinations, desires).

- Cognitive (feeling, imagination, memory and thinking).

- Volitional (emotions, feelings, moods, volitional processes).

- Communicative (nature and features of communication, interaction, interrelationship, interpersonal perception). 
A condition of rational behavior that includes the cognitive capabilities of the target audience, which allow it, in the absence of time and resource constraints, to obtain an idea of the significance of the likelihood of negative consequences of certain behavior.

The condition of individual choice is considered as unidirectional psychological influence on the target audience. Under this condition, the target audience is able to take additional steps to obtain information that is not available to the target audience.

The condition of complexity in its content is considered when the target audience is under constant psychological influence.

These conditions can be implemented through mass, group and individual communication means with using of different information transmission channels, and have a certain cycle of psychological influence.

The cycle of psychological influence consists of the following steps: attention attracting and interest creating; emotional stimulation; demonstration the way of how to remove the tension created.

Engaging and keeping the individual attention by the media is realized through regular and complex psychological influence. Complexity involves the following steps:

1. 1 step - passing the information about what is happening in the world (or regions);

2. 2 step - unification of society into a single whole (communicative-integration function);

3. 3 step - declaration the interests of society to those who govern or possess the power in the society ("voxpopuli" function);

4. 4 step - control or manipulation the behavior and instincts of society (population) by the power-holders, ruling class, state (political function);

5. 5 step - upbringing and partial education of the younger and older generations (function of socialization).

These steps of complex psychological influence reflect the content of general assumptions (the quantitative and qualitative characteristics of negative content or information are quite big or external and internal conditions of distribution are constantly changing). This creates an idea of a measure of probabilistic type, the physical content of which is the dynamics of changing the view of the target audience during some period of time about specific negative content or information. So, this indicator can be taken as a coefficient of effectiveness of psychological influence, and assume that its value tends to zero.

Substantially, this assumption means that the target audience's view of one or the other information is close to 0 or 1 . In this situation, it is difficult to change this (means stable thoughts), whereas ideas close to 0.5 change relatively easily (unstable thoughts).

Analysis of the above gives an opportunity to determine the parameters of the proposed model of evaluation of negative psychological influence.

The first parameter is introduction of the function of imagination, which may represent the subjective probability (the digital weight of the likelihood of particular person or target audiences believes that the information obtained will really change their behavior and have signs of psychological influence).

The implementation of this parameter is influenced by the sources through which the target audience receives information. Therefore, it would be advisable to use CobbDouglas-type functions to justify this parameter

$$
g_{i j}\left(r_{i}\right)=a_{i}\left(r_{i}\right)^{\lambda_{i}}\left(z_{i j}\right)^{\mu_{i j}},
$$

where: 
$g_{i j}$ - available to the target audience $\mathrm{j}$ information resource of a certain manipulative quality i;

$a_{i}$ - manipulative technology used;

$r_{i}$ - the cost of the subject $\mathrm{i}$ to produce the content;

$z_{i j}$ - the cost of subject $\mathrm{i}$ to deliver the negative content to the target audience;

$\lambda_{i}\left(\mu_{i j}\right) \geq 0$ - the coefficients of elasticity by factor $r_{i}\left(z_{i j}\right)$.

The means of delivering information (content) can be very different: television, radio, newspapers and magazines, websites and blogs, rumors etc. To be receptive, an information resource must be useful, that is, from the standpoint of journalism principles, provide, in addition to the information function, all other functions of the influencers, such as the media, organizations and leaders who have a certain reputation or trust among target audience.

The second parameter of the proposed model is the determination the information control tasks or manipulations, which can be divided into two types: the first - gaining the trust of the target audience and creating information superiority over other subjects of control; and the second - conducting the psychological operations in conditions of level of contact reached with target audience. The first type of tasks is long-term oriented. They include researches and classification of the target audience, identification of real and potential problems, analysis of the actions of other subjects of control.

Consider the possible formulation of the first type problem. Suppose there are $n$ target audiences and $m$ management subjects (centers) of control, $C_{i j} \geq 0$ is the worth of target audience $j(j=1, \ldots n)$ for the center $i(i=1, \ldots m)$, and $u_{i j} \geq 0$ is the usefulness of the information resource of center $i$ for the target audience $j$. Each negative information has a center and has a financial resource $\mathrm{R}$ when $i>0$. It is required to find the optimal financial resources allocation among the target audiences. If there is one center $(m=1)$, it is necessary to solve the following optimization problem (indexes i omitted):

$$
\begin{aligned}
& \sum_{j=1}^{n} c_{j} u_{j}\left(z_{j}\right)^{j} \rightarrow \max \\
& r_{j}+\sum_{j=1}^{n} z_{j} \leq R
\end{aligned}
$$

As a result of the solution of game methods for the first type of parameters, the distribution of information, which has the signs of negative psychological influence $G_{i j}$ on the target audiences, will be found. For each audience $j$, the degree of negative psychological impact of the subject $i$ is equal to

$$
K_{i j}=\frac{G_{i j}}{\sum_{k=1}^{m} G_{i j}} ; i=1, \ldots, m ; j=1, \ldots, n .
$$

Hence, the informational influence of subject $i$ on the audience $j$, that used as an input parameter in the models of information perception, as well 


$$
y_{i j}=Y_{O} K_{i j} ;=1, \ldots, m ; j=1, \ldots, n
$$

Thus, the model of information perception can be used in solving problems of information influence and control in various fields of practical activity. The calculated parameters of the proposed model are shown in Figure 5.

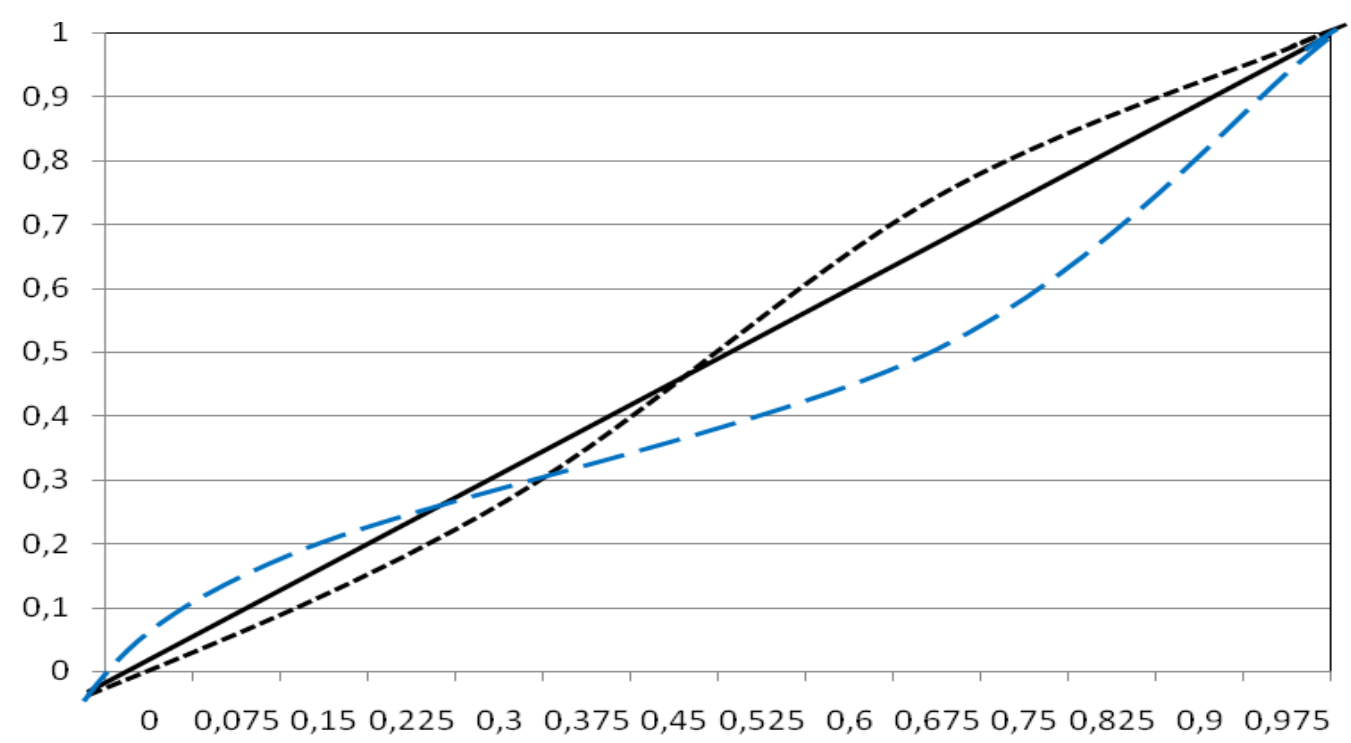

Figure 5. Parameters of the Information Perception Model

- _ - target audience's anticipated behavior indicators $\delta=0,98 ; \mathrm{y}=0,83$;

_ _ _ - target audience's multidirectional behavior indicators $\delta=0,77 ; \mathrm{y}=0,62$.

Figure 5 presents the parameter estimates calculated with using of the Wolfram Mathematica 7 package (FindFit function). Information flows are not organized during the experiment itself.

From the results of Figure 5 we can conclude: the individuals are in the conditions of multidirectional informational influences, and one of the influences, that aimed at reducing the impression of probability, is slightly stronger. Accordingly, the effect of subjects' overestimation of low probabilities and underestimation of high ones observed in the experiments can be explained by constant multidirectional information influences with approximately the same characteristics. Function graph at $\delta=0.77 ; \mathrm{y}=0.62$ has a stronger S-shaped bend than when $\delta=0.98 ; \mathrm{y}=0.83$ (Figure 5). This can be explained by the fact that individuals are influenced by multidirectional information flows of greater intensity. Of course, these information flows are not organized during the experiment itself. Subjects, while solving certain tasks, take into account their personal experience and their previously acquired knowledge and testimony.

Thus, expressions (1) - (5) reflect the multi-stage process of external information perception by subjects. Information pervasion into the consciousness of the individual is described by logistical dependence with the parameters of information inclusion in the world of consciousness of individuals, and depends on its orientation, characterized by the parameter.

Taking this into account is the basis of a methodology for forming a set of target audience behavior models, which is now quite simply can be implemented through 
scaling procedure during observation, that is, through the collection of information processes statistics and their processing not for the period $\Delta \mathrm{T}=1$ year, but for a much shorter period $\Delta \mathrm{t}(\Delta \mathrm{t} \ll \Delta \mathrm{T})$, for example, for a month or a week, due to the implementation of the algorithm:

$$
\chi_{s}=\chi \frac{\Delta T}{\Delta t}, \quad i=\overline{1,5},
$$

where the intensity $\chi$ is calculated by the period $\Delta \mathrm{t}<<\mathrm{T}$.

For example, if for $\Delta t=1$ week the number of 3000 points is obtained, then applying formula (4) we get:

$$
\chi \mathrm{s}=3000 \times 365 / 7 \approx 156,5 \times 103 \text { (points). }
$$

Such a number of points indicates that the level of negative psychological impact for one week is within the information background (see Figure 5) and does not actually affect the reduction of the moral and psychological status of the armed forces personnel.

If for $\Delta t=1$ week a score of 13500 points is obtained, then applying formula (4) we get:

$$
\chi \mathrm{s}=13500 \times 365 / 7 \approx 704 \times 103 \text { (points). }
$$

In this case, the level of negative psychological influence is within the limits of occurrence of influence (see Figure 5), during which the moral and psychological status of the armed forces personnel begins to deteriorate. Therefore, only in this range of values of the index $\chi s$ and above it, the fact of detection of negative influence (on the basis of the moral and psychological state of the troops) is fixed.

\section{Conclusion}

Thus, the proposed approach allows to simulate the intellectual behavior of target audiences and create the opportunity to quantify the level of psychological impact on a specific target audience over a specified period of time. This contributes to the functions fulfillment of the relevant military headquarter. The additional effects of modeling are: relatively objectively anticipation of possible changes in the situation, and responding (counteracting) to negative processes adequately and pre-emptively. Therefore, this approach should be considered as an integral element of the subsystem of monitoring the situation in the general outline of the system of counteracting the negative psychological influence on military personnel.

The proposed methodological approach is universal in nature and can therefore be applied in the development of similar methodological tools, not only for the military sphere, but for the entire social system of the state or its sectors in the interests of ensuring internal political stability. Further research envisages the process of verification of the developed methodology, as well as the substantiation of proposals on ways to put into practice the structural units of the security and defense sector of the state.

\section{References}

[1] Kornev M.N. Social psychology: a textbook / M.N. Kornev, A.B. Kovalenko. - K .: 1995. - 304 p.

[2] Information-psychological and psychotronic war: a textbook / Under gen. ed. A.E. Taras. - Mn.: Harvest, 2003. - 432 p.

[3] Katsalap V.O., Voitko O.V., Rakhimov V.V. Analysis of the features of manipulation as a tool of psychological influence on consciousness / V.O. Katsalap, O.V. Voitko, V.V. Rakhimov // Scientific journal "Modern information technologies in the field of security and defense" K .: NDUU. - 2019. - №2 (35). - P. 121-126. 
[4] Katsalap V.O., Voitko O.V., Tsurko Y.V. Methodical approach to identification of sources of threats to information security in the military sphere / V.O. Katsalap, O.V. Wojtko, Y.V. Tsurko // Scientific journal "Modern information technologies in the field of security and defense" K .: NDUU. - 2019. - №1 (34). - P. 103-108.

[5] Katsalap V.O., Voitko O.V., Chernega V.M. Methods of assessment of threats to information security of Ukraine in the military sphere / V.O. Katsalap, O.V. Voitko, V.M. Cherenga // Scientific journal "Modern information technologies in the field of security and defense" K .: NDUU. - 2018. - №1 (31). P. 149-154.

[6] Katsalap V.O., Voitko O.V. Evaluation of information and psychological impact in the interests of hostilities (VO) / V.O. Katsalap, O.V. Voitko // Scientific journal "Modern information technologies in the field of security and defense" K .: NDUU. - 2017. - №2 (29). - P. 116-120. 